\title{
Suppression of Rice Stripe Virus Replication in Laodelphax striatellus Using Vector Insect-Derived Double-Stranded RNAs
}

\author{
Ying Fang ${ }^{1}$, Jae Young Choi ${ }^{1}$, Dong Hwan Park ${ }^{1}$, Min Gu Park ${ }^{1}$, Jun Young Kim ${ }^{1}$, Minghui Wang ${ }^{1}$, \\ Hyun Ji Kim ${ }^{1}$, Woo Jin Kim ${ }^{1}$, and Yeon Ho Je ${ }^{1,2 *}$ \\ ${ }^{I}$ Department of Agricultural Biotechnology, College of Agriculture \& Life Science, Seoul National University, Seoul \\ 08826, Korea \\ ${ }^{2}$ Research Institute for Agriculture and Life Sciences, Seoul National University, Seoul 08826, Korea
}

(Received on March 13, 2020; Revised on May 6, 2020; Accepted on May 6, 2020)

RNA interference (RNAi) has attracted attention as a promising approach to control plant viruses in their insect vectors. In the present study, to suppress replication of the rice stripe virus (RSV) in its vector, Laodelphax striatellus, using RNAi, dsRNAs against $L$. striatellus genes that are strongly upregulated upon RSV infection were delivered through a rice leaf-mediated method. RNAi-based silencing of peroxiredoxin, cathepsin $B$, and cytochrome $P 450$ resulted in significant down regulation of the NS3 gene of RSV, achieving a transcriptional reduction greater than $73.6 \%$ at a concentration of $100 \mathrm{ng} / \mu \mathrm{l}$ and, possibly compromising viral replication. $L$. striatellus genes might play crucial roles in the transmission of RSV; transcriptional silencing of these genes could suppress viral replication in $L$. striatellus. These results suggest effective RNAi-based approaches for controlling RSV and provide insight into RSV-L. striatellus interactions.

Keywords : double-stranded RNA, Laodelphax striatellus, rice stripe virus, RNA interference

Handling Editor : Jungkwan Lee

\footnotetext{
*Corresponding author.

Phone) +82-2-880-4706, FAX) +82-2-873-2319

E-mail)btrus@snu.ac.kr

(c) This is an Open Access article distributed under the terms of the Creative Commons Attribution Non-Commercial License (http:// creativecommons.org/licenses/by-nc/4.0) which permits unrestricted noncommercial use, distribution, and reproduction in any medium, provided the original work is properly cited.
}

Articles can be freely viewed online at www.ppjonline.org.
Rice stripe virus (RSV) is one of the most damaging plant viruses and cause significant reductions of rice yield in temperate and subtropical regions including Korea, China, and Japan (Wei et al., 2009). RSV-infected rice shows chlorotic stripes or necrotic streaks on newly developed leaves and subsequently exhibits premature wilting and stunted growth (Hibino, 1996). RSV is a non-enveloped virus with a genome of four negative-sense or ambisense segments of single-stranded RNA (Hamamatsu et al., 1993; Ishikawa et al., 1989). RSV is transovarially transmitted in a persistent manner by Laodelphax striatellus (Toriyama, 1986). Specific interactions between L. striatellus and RSV are required for successful transmission of RSV; it has been reported that healthy rice could not be infected with RSV extracted from viruliferous rice (Zhao et al., 2016b). Transcriptome and proteome-based comparative analyses of RSV-viruliferous and non-viruliferous $L$. striatellus have been performed to elucidate the interactions between RSV and L. striatellus (Lee et al., 2013; Liu et al., 2015; Liu et al., 2016; Zhao et al., 2016a).

RNA interference (RNAi) is considered as a promosing strategy for interfering with the replication of plant viruses in their vector insects (Kanakala and Ghanim, 2016), as it is an important mechanism of defense against viral infection in plants and insects (Stram and Kuzntzova, 2006; Wang et al., 2006). In insects, RNAi-mediated antiviral immunity involves cleavage of double-stranded RNAs (dsRNAs) or short hairpin RNAs (shRNAs) into virus-derived small interfering RNAs (vsiRNAs) by the RNase III enzyme, Dicer (Galiana-Arnoux et al., 2006; Xu et al., 2012). These vsiRNAs are assembled into the RNA-induced silencing complex by interaction with Argonaute 2 (AGO2) to cleave complementary mRNAs (Van Rij et al., 2006).

RNAi-mediated control of plant viruses in their vector 
insects could be achieved either directly by silencing of viral transcripts or indirectly by silencing host genes essential for viral replication (Kanakala and Ghanim, 2016). Replication of RSV in L. striatellus has been efficiently suppressed not only by application of exogenous RSV-specific dsRNAs (An et al., 2017) but also by transcriptional silencing of $L$. striatellus genes, specifically, CRP1 and LsE75 (Fang et al., 2017; Liu et al., 2015). In a previous comparative transcriptomic analysis, we identified $L$. striatellus genes that are strongly upregulated in RSV-viruliferous L. striatellus (Lee et al., 2013). In the present study, we investigated the efficacy of RNAinduced transcriptional silencing of $L$. striatellus genes that are upregulated in RSV-viruliferous $L$. striatellus in suppressing RSV replication in this vector.

\section{Materials and Methods}

Insect and virus. Individual of non-viruliferous $L$. striatellus were collected from the healthy rice (Oryza sativa) in the field and reared on uninfected rice in an insectary at $28^{\circ} \mathrm{C}$ and $80 \%$ relative humidity under a $16 \mathrm{~h}$ light $/ 8 \mathrm{~h}$ dark photocycle. The RSV-infested rice used in this study was kindly provided by the National Institute of Crop Science, Miryang, Korea. RSV-viruliferous L. striatellus was obtained by feeding 2 nd instar nymphs of naive $L$. striatellus on RSV-infected rice (5-6 cm tall) for 5 days, with RSV infection confirmed by reverse transcription polymerase chain reaction as previously described (An et al., 2017). The RSV-viruliferous L. striatellus individuals were provided RSV-infested rice periodically and maintained in the laboratory at $28^{\circ} \mathrm{C}$ and $80 \%$ relative humidity under a $16 \mathrm{~h}$ light $/ 8 \mathrm{~h}$ dark photocycle.

Combined transcriptome analysis. The short sequence reads of RSV-viruliferous and non-viruliferous $L$. striatellus that had previously been submitted to the NABIC (National Agriculture Biotechnology Information Center, Rural Development Administration, Korea) nextgeneration sequencing (NGS) Sequence Read Archive (SRA) database under accession numbers NN-0890 and NN-0884 were pooled and filtered using NGS QC Toolkit v2.3 (Patel and Jain, 2012) to remove low quality (Q-score $>30$ ) sequence reads. Trinity de novo assembler v2.4 Software (Grabherr et al., 2011) were then used to generate contigs longer than $200 \mathrm{bp}$ from the filtered clean reads. The Trimmomatic (Bolger et al., 2014) command line option of Trinity software was applied to remove adapter and artifact sequences from the quality-filtered short reads. The standalone version of the TransDecoder program was used to predict protein-coding sequences, Subsequently, the CD-HIT-EST program was used to cluster homologous sequences with default parameters to merge highly homologous contigs (identity $>95 \%$ ) to construct the final version of in silico cDNA library. The filtered short reads were then mapped to the cDNA library sequences using the Bowtie2 program (Langmead and Salzberg, 2012) with default parameters. The mapping results of each sample obtained by Bowtie2 were then quantified using the eXpress program (Roberts and Pachter, 2013). The digital gene expression profiles of each sample obtained by eXpress were generated as fragments per kilobase of exon model per million fragments mapped (FPKM) values (Mortazavi et al., 2008) to compare the gene expression levels between RSV-viruliferous and non-viruliferous $L$. striatellus.

Synthesis of dsRNA. Candidate siRNA sites in target genes were predicted using BLOCK-iT RNAi Designer (https://rnaidesigner.thermofisher.com/rnaiexpress) and dsRNA sequences 500-700 bp in length and containing at least three candidate sites of siRNA were designed against each target gene. Single-strand cDNA of each target gene was synthesized from total RNA of $L$. striatellus using the QuantiTect Reverse Transcription Kit (Qiagen, Hilden, Germany) according to manufacturer's instructions and the target gene was amplified using KOD Neo FX DNA polymerase (Toyobo, Osaka, Japan) and primers with a T7 promoter sequence (5'-TAATACGACTCACTATAG-3') at the 5'-end (Supplementary Table 1) as previously described (Fang et al., 2017). Using the amplified product as template, dsRNA for the target gene was produced by Genolution Phamaceuticals (Seoul, Korea).

RNAi in L. striatellus. A series of concentrations (10, 50, and $250 \mathrm{ng} / \mu \mathrm{l}$ in $10 \%$ sucrose solution) of each dsRNA was applied to 4 th instar nymphs of $L$. striatellus by a ricemediated feeding method as previously described (An et al., 2017). Briefly, a feeding chamber was fabricated from a 15-ml conical tube (Fisher Scientific, Waltham, MA, USA) with a hole at the top to provide air; a $10-\mu 1$ tip was inserted in the hole to prevent $L$. striatellus individuals from escaping the tube. Parafilm M film (Bemis, Oshkosh, WI, USA) was used to seal the gap between 15-ml tube serving as the feeding chamber and the tube cap to create a reservoir for dsRNA solution. Three hundred microliters of each dsRNA solution was dispensed into the reservoir, and 3 rice leaves were placed over the dsRNA solution. Fifteen 4th instar nymphs of $L$. striatellus were treated using the 3 dsRNA-permeated rice leaves in the feeding chamber 
and placed in a growth chamber with a temperature of $28^{\circ} \mathrm{C}, 80 \%$ relative humidity, and a $16 \mathrm{~h}$ light $/ 8 \mathrm{~h}$ dark photocycle. Total RNA was extracted from the dsRNAfed nymphs at $48 \mathrm{~h}$ post-treatment using QIAzol Lysis Reagent (Qiagen) following the manufacturer's instructions. cDNA synthesis and quantitative PCR (qPCR) using the primers listed in Supplementary Table 2 were conducted as previously described (Fang et al., 2017). All experiments were performed in triplicate on different days.

Statistical analysis. Statistical analysis was performed by one-way ANOVA using SPSS Statistics version 24 (IBM Corp., Armonk, NY, USA). Multiple comparisons of mean values were performed by post hoc Scheffé's tests. $P$-values less than 0.05 were considered statistically significant.

\section{Results}

Comparative transcriptome analysis. To investigate genes in L. striatellus that were differentially expressed upon RSV infection, total raw reads from the RSV- viruliferous and non-viruliferous $L$. striatellus individuals were mapped to the 3,703 contig sequences that were functionally categorized in the gene ontology (GO) analysis to obtain the FPKM values. While 885 genes were over two-fold up-regulated in RSV-viruliferous $L$. striatellus relative to non-viruliferous $L$. striatellus, only 69 genes were over two-fold down-regulated in the former relative to the latter (Table 1). In particular, genes classified into the GO subcategories: [Q] secondary metabolites biosynthesis, transport and catabolism, $[\mathrm{P}]$ inorganic ion transport and metabolism, [I] lipid transport and metabolism, and [Z] cytoskeleton were expressed uniquely among the two-fold differentially expressed genes.

Selection and validation of target genes for RNAi application. To investigate whether RSV transmission in L. striatellus could be suppressed by silencing host genes related to RSV transmission, we selected 8 genes identified as up-regulated in the RSV-viruliferous $L$. striatellus based on the comparative transcriptome analysis and qPCR validation of relative transcription level (Table 2). Among

Table 1. Differentially expressed genes in RSV-viruliferous Laodelphax striatellus

\begin{tabular}{|c|c|c|c|}
\hline Functional group & Sub-category & $\begin{array}{l}\text { No. of genes down- } \\
\text { regulated over 2-fold }\end{array}$ & $\begin{array}{l}\text { No. of genes up- } \\
\text { regulated over 2-fold }\end{array}$ \\
\hline \multirow[t]{8}{*}{ Metabolism } & [Q] Secondary metabolites biosynthesis, transport and catabolism & 2 & 50 \\
\hline & {$[\mathrm{P}]$ Inorganic ion transport and metabolism } & 0 & 43 \\
\hline & [I] Lipid transport and metabolism & 13 & 66 \\
\hline & {$[\mathrm{H}]$ Coenzyme transport and metabolism } & 0 & 17 \\
\hline & [F] Nucleotide transport and metabolism & 1 & 10 \\
\hline & [E] Amino acid transport and metabolism & 3 & 54 \\
\hline & [G] Carbohydrate transport and metabolism & 3 & 64 \\
\hline & [C] Energy production and conversion & 3 & 72 \\
\hline \multirow{10}{*}{$\begin{array}{l}\text { Cellular processes } \\
\text { and signaling }\end{array}$} & [O] Posttranslational modification, protein turnover, chaperones & 8 & 142 \\
\hline & [U] Intracellular trafficking, secretion, and vesicular transport & 1 & 32 \\
\hline & [W] Extracellular structures & 0 & 4 \\
\hline & [Z] Cytoskeleton & 3 & 79 \\
\hline & {$[\mathrm{N}]$ Cell motility } & 0 & 0 \\
\hline & [M] Cell wall, membrane, envelope biogenesis & 0 & 20 \\
\hline & [T] Signal transduction mechanisms & 4 & 104 \\
\hline & [V] Defense mechanisms & 1 & 4 \\
\hline & [Y] Nuclear structure & 0 & 2 \\
\hline & [D] Cell cycle control, cell division, chromosome partitioning & 3 & 5 \\
\hline \multirow{5}{*}{$\begin{array}{l}\text { Information } \\
\text { storage and } \\
\text { processing }\end{array}$} & [B] Chromatin structure and dynamics & 8 & 8 \\
\hline & [L] Replication, recombination and repair & 1 & 1 \\
\hline & {$[\mathrm{K}]$ Transcription } & 2 & 45 \\
\hline & [A] RNA processing and modification & 9 & 33 \\
\hline & {$[\mathrm{J}]$ Translation, ribosomal structure and biogenesis } & 4 & 30 \\
\hline Total & & 69 & 885 \\
\hline
\end{tabular}

$\mathrm{RSV}$, rice stripe virus. 
Table 2. List of genes selected for RNAi application to suppress RSV replication in Laodelphax striatellus

\begin{tabular}{llccc}
\hline $\begin{array}{c}\text { Contig } \\
\text { no. }\end{array}$ & \multicolumn{1}{c}{ Blast hit } & $\begin{array}{c}\text { FPKM value } \\
\text { in RSV-viruliferous } \\
\text { L. striatellus }\end{array}$ & $\begin{array}{c}\text { FPKM value } \\
\text { in non-viruliferous } \\
\text { L. striatellus }\end{array}$ & $\begin{array}{c}\text { Up-regulated ratio in } \\
\text { RSV-viruliferous } \\
\text { L. striatellus }\end{array}$ \\
\hline 75761 & Structural constituent of cuticle protein & 159.90 & 36.15 & 4.42 \\
76485 & Actins & 442.70 & 97.24 & 4.55 \\
78749 & Peroxiredoxins, prx-1, prx-2, prx-3 protein & 0.91 & 0.57 & 1.59 \\
80690 & Host cell factor & 0.55 & 0.53 & 1.05 \\
81684 & Cathepsin B protein & 298.80 & 22.47 & 13.30 \\
82635 & ND & 9.90 & 2.74 & 3.61 \\
85613 & Cytochrome P450 protein & 4.76 & 1.43 & 3.33 \\
86923 & Protein involved in cellular iron ion & 420.20 & 46.32 & 9.07 \\
& homeostasis & & & \\
\hline
\end{tabular}

RNAi, RNA interference; RSV, rice stripe virus; FPKM, fragments per kilobase of exon model per million fragments mapped.

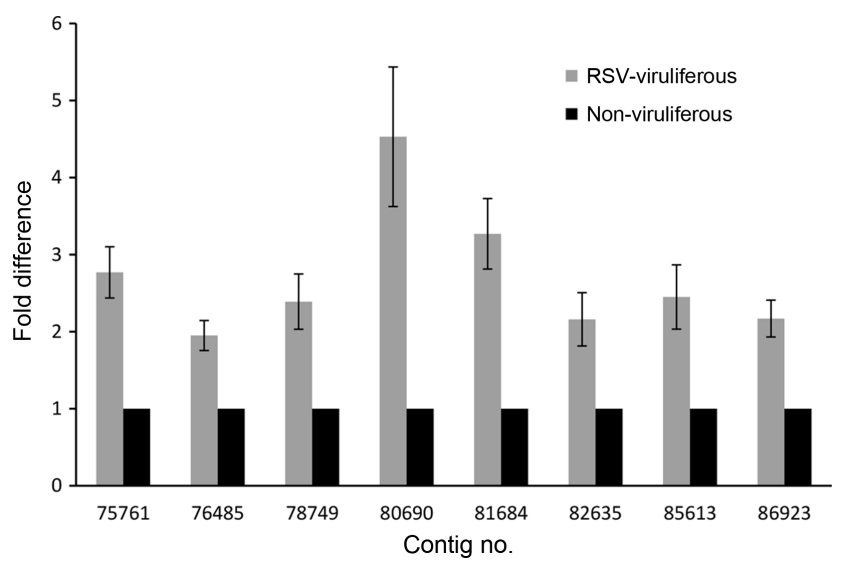

Fig. 1. Quantitative PCR (qPCR) analysis of the transcription of Laodelphax striatellus genes up-regulated in rice stripe virus (RSV)-viruliferous L. striatellus. The relative transcription levels are expressed as fold differences in RSV-viruliferous $L$. striatellus relative to non-viruliferous $L$. striatellus as determined by qPCR and calculated using the $2^{-\Delta \Delta \mathrm{Ct}}$ method.

these RNAi target genes, 6 genes (all except contig nos. 78749 and 80690) were shown to be at least 2-fold upregulated in the RSV-viruliferous $L$. striatellus in both the transcriptome sequencing and qPCR analyses (Fig. 1). The other 2 target genes, contig nos. 78749 and 80690, were shown to be highly up-regulated in the RSV-viruliferous L. striatellus in the qPCR analysis (2.39- and 4.53-fold up-regulated, respectively) (Fig. 1), although they were found to be only slightly up-regulated in the transcriptome sequencing analysis (Table 2).

Silencing of RNAi target genes in $L$. striatellus nymphs by feeding dsRNA. To evaluate the efficacy of dsRNAs in silencing the transcription of target genes potentially responsible for RSV replication in L. striatellus, each dsRNA was administered to RSV-viruliferous $L$. striatellus nymphs via the RNAi feeding system. qPCR revealed that transcription of all target genes tested was significantly decreased in each dsRNA-administered L. striatellus individual relative to the corresponding levels in control nymphs (Fig. 2). Among the 8 target genes, contig no. 78749 exhibited the greatest decrease in transcription (approximately 21-fold) upon treatment with the corresponding dsRNA .

Suppression of RSV replication in $L$. striatellus nymphs that ingested vector-derived dsRNAs. To investigate whether dsRNAs against $L$. striatellus genes up-regulated in RSV-viruliferous L. striatellus suppress replication of RSV in L. striatellus, qPCR analysis of the transcription of RSV genes in RSV-viruliferous L. striatellus individuals that had ingested dsRNA was performed. The transcription level of NS3, which is crucial for RSV replication and act as a viral suppressor of RNAi, was significantly reduced in L. striatellus individuals that had been treated with dsRNAs against contig nos. 76485 (ds76485), 78749 (ds78749), 80690 (ds80690), 81684 (ds81684), 82635 (ds82635), and 85613 (ds85613) compared with those treated with no dsRNA and dsGFP (Fig. 3). Notably, transcription of NS3 showed pronounced and concentration-dependent decreases in ds78749-, ds81684-, and ds85613-treated $L$. striatellus (Fig. 4).

\section{Discussion}

Plant-to-plant transmission of most plant viruses is mediated by vector insects in a non-persistent, semipersistent, or persistent manner (Ng and Falk, 2006). RSV 

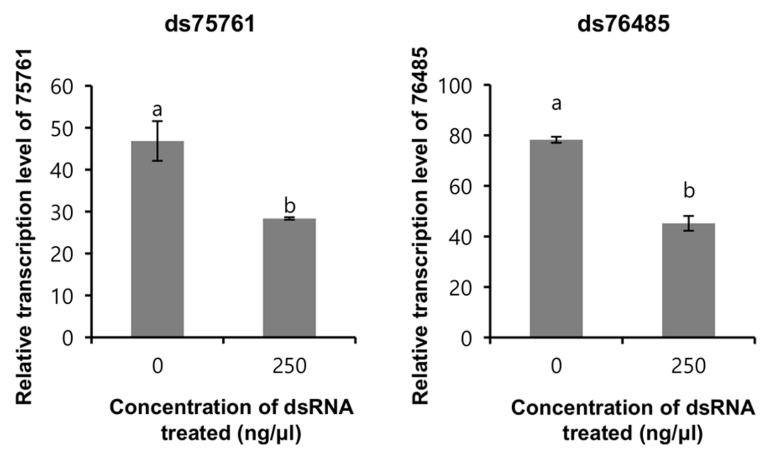

treated (ng/ $\mu \mathrm{l})$

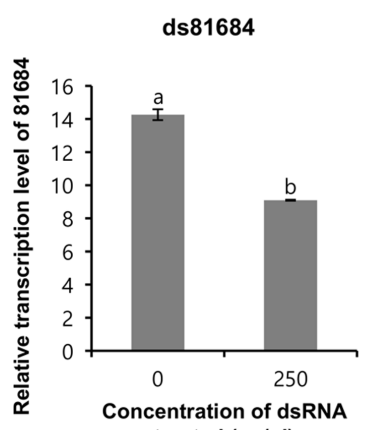

treated $(\mathrm{ng} / \mu \mathrm{l})$

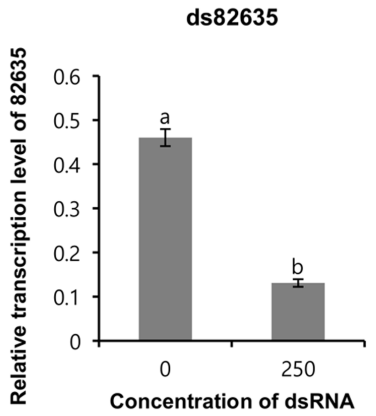

treated $(\mathrm{ng} / \mu \mathrm{l})$
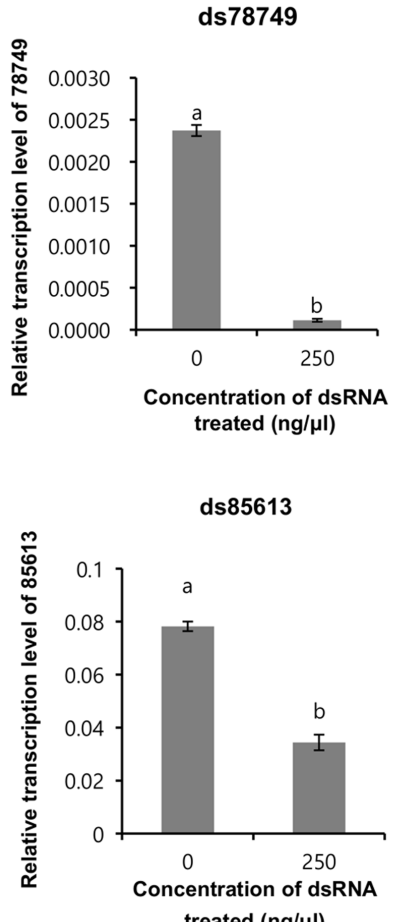

treated $(\mathrm{ng} / \mu \mathrm{l})$
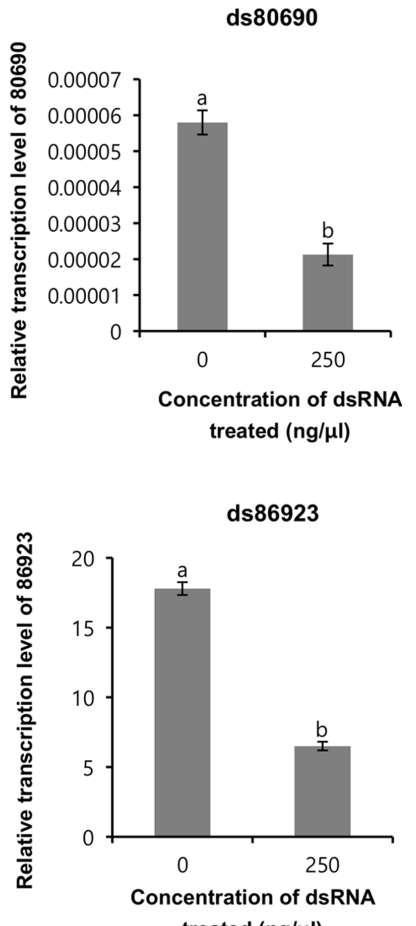

treated $(\mathrm{ng} / \mu \mathrm{l})$

Fig. 2. Silencing of RNA interference target gene transcription in rice stripe virus (RSV)-viruliferous Laodelphax striatellus nymphs that had ingested the corresponding double-stranded RNA (dsRNA). Fifteen 4th instar nymphs of RSV-viruliferous L. striatellus were fed on rice leaves steeped in each dsRNA for $48 \mathrm{~h}$. Relative transcription levels of each gene in RSV-viruliferous $L$. striatellus nymphs that had ingested the corresponding dsRNA were measured by quantitative PCR. Different letters above error bars (indicating \pm standard deviations) indicate a significant difference by post hoc tests $(P<0.05)$.

persistently replicates in the ovary of $L$. striatellus for transovarial transmission of the virus to the insect offspring and proliferates in the salivary glands for insect-to-plant transmission (Hogenhout et al., 2008; Wu et al., 2014). Replication of persistent viruses in their vector insects triggers antiviral immune defense mechanisms, including activation of the RNAi pathway (de Haro et al., 2017). In RSV-viruliferous L. striatellus, vsiRNAs involved in RNAi-based antiviral immunity could potentially regulate transcription of vector genes, and thereby alter biological processes in vector insects to support viral replication (Yang et al., 2018).

Recently, RNAi has been widely applied for knockdown of plant viruses in vector insects by silencing vector genes either to induce mortality of vector insects or to interfere with viral replication in vector insects (Kanakala and Ghanim, 2016). Although there are many reports of successful RNAi-based gene silencing to induce mortality of insect vectors, there are few reports regarding the silencing of insect vector genes to interfere plant virus replication in vector insects. In L. striatellus, silencing of two genes encoding cuticular protein (CRP1) and E75 nuclear receptor (LsE75) has been reported to suppress replication of RSV (Fang et al., 2017; Liu et al., 2015).

Several L. striatellus genes that are differentially expressed between RSV-viruliferous and non-viruliferous individuals have been identified through comparative transcriptome surveys (Lee et al., 2013). It has been speculated that strongly up-regulated genes in RSVviruliferous $L$. striatellus might play crucial roles in the replication of RSV and represent candidates for RNAibased silencing to suppress RSV replication in $L$. striatellus. Comparative transcriptome analysis revealed 8 genes that were up-regulated in RSV-viruliferous L. striatellus and that belonged to a diversity of GO groups. These 8 genes were selected for RNAi application to suppress replication of RSV in L. striatellus (Fig. 1). Among these selected genes, 4 genes annotated to peroxiredoxin (contig no. 78749), host cell factor (contig no. 80690), cathepsin B (contig no. 81684), and cytochrome P450 (contig no. 85613) have been previously reported to be up-regulated in an organ-specific manner in RSV infection (Zhao et al., 2016a). In this study, exogenous application of dsRNA against these 4 genes resulted in significant suppression of 


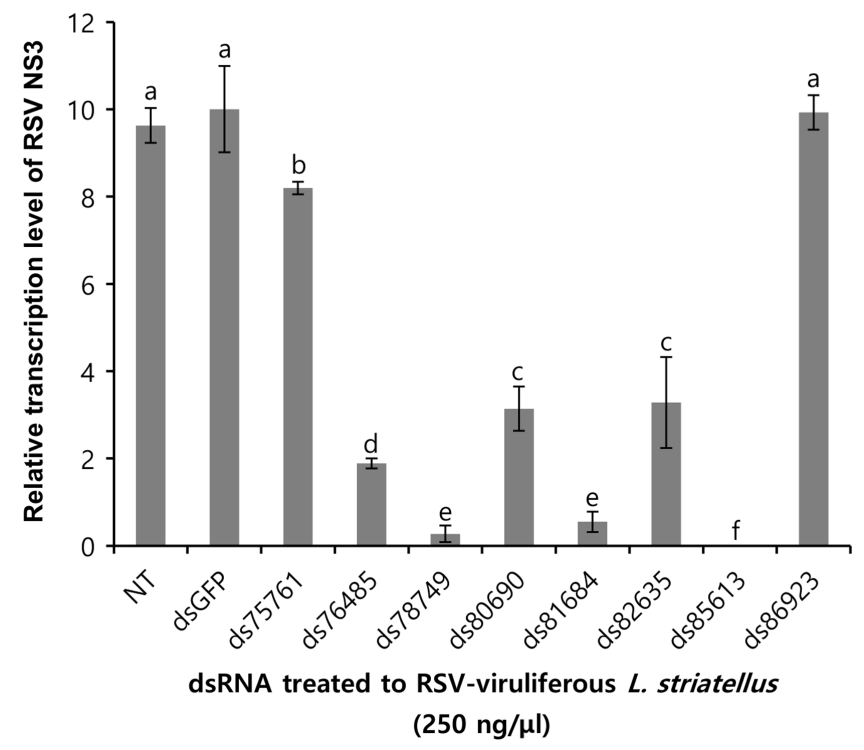

Fig. 3. Suppression of rice stripe virus (RSV) transmission in RSV-viruliferous Laodelphax striatellus nymphs that had ingested vector-derived double-stranded RNAs (dsRNAs). Fifteen 4th instar nymphs of RSV-viruliferous $L$. striatellus were fed on rice leaves steeped in each dsRNA ( $250 \mathrm{ng} / \mu \mathrm{l})$ for $48 \mathrm{~h}$. NT indicates RSV-viruliferous L. striatellus nymphs that had not ingested dsRNA. Relative transcription levels of the RSV NS3 gene in $L$. striatellus nymphs that had ingested the vector-derived dsRNAs were measured by quantitative PCR. Different letters above error bars (indicating \pm standard deviations) indicate a significant difference by post hoc tests $(P<0.05)$.
RSV replication in L. striatellus (Fig. 3). Transcriptional silencing of peroxiredoxin, cathepsin B, and cytochrome P450 at very low concentration of dsRNA (as low as 0.4 $\mathrm{ng} / \mu \mathrm{l}$ ) markedly suppressed replication of RSV (Fig. 4). These results demonstrated that comparative transcriptome analysis can aid the identification of genes related to the transmission of plant viruses in vector insects and that vector insect-derived genes related to replication of plant viruses could be effectively exploited to control plant viruses.

Peroxiredoxin is a ubiquitous family of antioxidant enzymes that eliminate reactive oxygen species that induce damage to host DNA, proteins, and lipid membranes, and cause dysfunction of cytoplasmic and nuclear signaling (Storr et al., 2013). In Bombyx mori, peoxiredoxin has been reported to potentially play a protective role against oxidative stress caused by viral infection (Lee et al., 2005). This finding suggests that post-transcriptional silencing of peoxiredoxin in L. striatellus might negativley affect RSV replication by hindering protection of $L$. striatellus from oxidative damage caused by RSV infection.

Cathepsin B is a lysosomal cysteine protease that play a crucial role in intracellular proteolysis (Premzl et al., 2006). In insects, cathepsin B is involved in various physiological processes, including silk gland histolysis during metamorphosis of $B$. mori larvae and yolk protein degradation during embryogenesis and adult fat body decomposi-

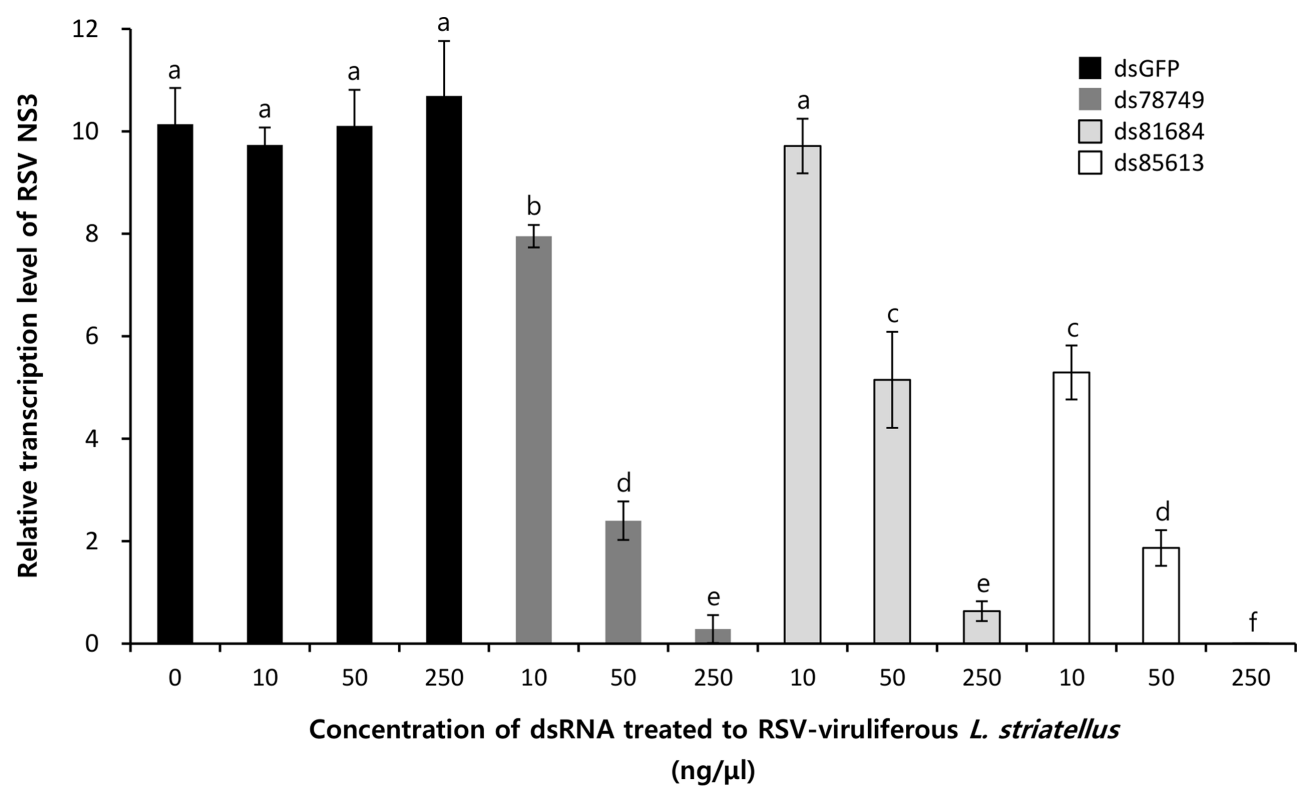

Fig. 4. Dose-dependent suppression of rice stripe virus (RSV) transmission in RSV-viruliferous Laodelphax striatellus nymphs that had ingested vector-derived double-stranded RNAs (dsRNAs). Fifteen 4th instar nymphs of RSV-viruliferous $L$. striatellus were fed rice leaves steeped in various concentrations of each dsRNA $(10,50$, and $250 \mathrm{ng} / \mu \mathrm{l})$ for $48 \mathrm{~h}$. Relative transcription levels of the RSV NS3 gene in L. striatellus nymphs fed the vector-derived dsRNAs were measured by quantitative PCR. Different letters above error bars (indicating \pm standard deviations) indicate a significant difference by post hoc tests $(P<0.05)$. 
tion of Helicoverpa armigera (Shiba et al., 2001; Yang et al., 2006; Zhao et al., 2005). RNAi-mediated silencing of cathepsin B gene was found to significantly reduce replication of dengue virus in the salivary gland of Aedes aegypti (Sim et al., 2012), consistent with the results of the present study. Because cathepsin B has been reported to trigger apoptosis (Jäättelä and Tschopp, 2003; Zuzarte-Luis et al., 2007), it was hypothesized that inhibition of apoptosis by silencing of cathepsin B would preserve dengue virus-infected cells, maintaining dsRNA production and impairing replication of the virus through RNAi (Sim et al., 2012). On the other hand, cathepsin B was found to be involved in decreased transmission efficiency of the potato leaf roll virus (PLRV) in Myzus persicae (Pinheiro et al., 2017). These observations indicate that relationships between cathepsin B and plant viruses in insect vectors are complex and vary among plant viruses and vector insects.

Cytochrome $\mathrm{P} 450$ proteins are a superfamily of hemethiolate enzymes that have been found in eukaryotes, archaea, bacteria, and viruses (Lamb et al., 2009). Insect genomes encode numerous, diverse cytochrome P450s which contribute to diverse physiological roles including metabolism of ecdysteroids and juvenile hormones, cuticular hydrocarbon synthesis, and protection against xenobiotics (Feyereisen, 2015). In addition, several cytochrome P450s are critical for insecticide resistance (David et al., 2013). Although many examples of cytochrome P450-mediated insecticide resistance have been reported in insect vectors of plant viruses, including L. striatellus, Nilaparvata lugens, and Trialeurodes vaporariorum (Bass et al., 2011; Elzaki et al., 2016; Karatolos et al., 2012), relationships between cytochrome P450s and plant viruses have not been described. Further studies are required to explore the effects of cytochrome P450s on the replication of RSV in L. striatellus, which might provide further insights into RSV-L. striatellus interactions.

\section{Acknowledgments}

This work was carried out with the support of "Cooperative Research Program for Agriculture Science and Technology Development (Project No. PJ01497803)" Rural Development Administration, Republic of Korea. Y. Fang, D. H. Park, M. G. Park, J. Y. Kim, M. Wang, and H. J. Kim were supported by the Brain Korea 21 plus project, Seoul National University, Republic of Korea.

\section{Electronic Supplementary Material}

Supplementary materials are available at The Plant Pathol- ogy Journal website (http://www.ppjonline.org/).

\section{References}

An, S. B., Choi, J. Y., Lee, S. H., Fang, Y., Kim, J. H., Park, D. H., Park, M. G., Woo, R. M., Kim, W. J. and Je, Y. H. 2017. Silencing of rice stripe virus in Laodelphax striatellus using virus-derived double-stranded RNAs. J. Asia-Pac. Entomol. 20:695-698.

Bass, C., Carvalho, R. A., Oliphant, L., Puinean, A. M., Field, L. M., Nauen, R., Williamson, M. S., Moores, G. and Gorman, K. 2011. Overexpression of a cytochrome P450 monooxygenase, CYP6ER1, is associated with resistance to imidacloprid in the brown planthopper, Nilaparvata lugens. Insect Mol. Biol. 20:763-773.

Bolger, A. M., Lohse, M. and Usadel, B. 2014. Trimmomatic: a flexible trimmer for Illumina sequence data. Bioinformatics 30:2114-2120.

David, J.-P., Ismail, H. M., Chandor-Proust, A. and Paine, M. J. I. 2013. Role of cytochrome P450s in insecticide resistance: impact on the control of mosquito-borne diseases and use of insecticides on Earth. Philos. Trans. R. Soc. Lond. B Biol. Sci. 368:20120429.

de Haro, L. A., Dumón, A. D., Mattio, M. F., Argüello Caro, E. B., Llauger, G., Zavallo, D., Blanc, H., Mongelli, V. C., Truol, G., Saleh, M.-C., Asurmendi, S. and del Vas, M. 2017. Mal de Rio Cuarto virus infection triggers the production of distinctive viral-derived siRNA profiles in wheat and its planthopper vector. Front. Plant Sci. 8:766.

Elzaki, M. E. A., Zhang, W., Feng, A., Qiou, X., Zhao, W. and Han, Z. 2016. Constitutive overexpression of cytochrome P450 associated with imidacloprid resistance in Laodelphax striatellus (Fallén). Pest Manag. Sci. 72:1051-1058.

Fang, Y., Choi, J. Y., Lee, S. H., Kim, J. H., Park, D. H., Park, M. G., Woo, R. M., Lee, B. R., Kim, W. J., Li, S. and Je, Y. H. 2017. RNA interference of E75 nuclear receptor gene suppresses transmission of rice stripe virus in Laodelphax striatellus. J. Asia-Pac. Entomol. 20:1140-1144.

Feyereisen, R. 2015. Insect P450 inhibitors and insecticides: challenges and opportunities. Pest Manag. Sci. 71:793-800.

Galiana-Arnoux, D., Dostert, C., Schneemann, A., Hoffmann, J. A. and Imler, J.-L. 2006. Essential function in vivo for Dicer-2 in host defense against RNA viruses in drosophila. Nat. Immunol. 7:590-597.

Grabherr, M. G., Haas, B. J., Yassour, M., Levin, J. Z., Thompson, D. A., Amit, I., Adiconis, X., Fan, L., Raychowdhury, R., Zeng, Q., Chen, Z., Mauceli, E., Hacohen, N., Gnirke, A., Rhind, N., di Palma, F., Birren, B. W., Nusbaum, C., Lindblad-Toh, K., Friedman, N. and Regev, A. 2011. Fulllength transcriptome assembly from RNA-Seq data without a reference genome. Nat. Biotechnol. 29:644-652.

Hamamatsu, C., Toriyama, S., Toyoda, T. and Ishihama, A. 1993. Ambisense coding strategy of the rice stripe virus genome: in vitro translation studies. J. Gen. Virol. 74:1125-1131. 
Hibino, H. 1996. Biology and epidemiology of rice viruses. Annu. Rev. Phytopathol. 34:249-274.

Hogenhout, S. A., Ammar, E.-D., Whitfield, A. E. and Redinbaugh, M. G. 2008. Insect vector interactions with persistently transmitted viruses. Annu. Rev. Phytopathol. 46:327-359.

Ishikawa, K., Omura, T. and Hibino, H. 1989. Morphological characteristics of rice stripe virus. J. Gen. Virol. 70:34653468.

Jäättelä, M. and Tschopp, J. 2003. Caspase-independent cell death in T lymphocytes. Nat. Immunol. 4:416-423.

Kanakala, S. and Ghanim, M. 2016. RNA interference in insect vectors for plant viruses. Viruses 8:329.

Karatolos, N., Williamson, M. S., Denholm, I., Gorman, K., Ffrench-Constant, R. H. and Bass, C. 2012. Over-expression of a cytochrome $\mathrm{P} 450$ is associated with resistance to pyriproxyfen in the greenhouse whitefly Trialeurodes vaporariorum. PLoS ONE 7:e31077.

Lamb, D. C., Lei, L., Warrilow, A. G. S., Lepesheva, G. I., Mullins, J. G. L., Waterman, M. R. and Kelly, S. L. 2009. The first virally encoded cytochrome p450. J. Virol. 83:8266-8269.

Langmead, B. and Salzberg, S. L. 2012. Fast gapped-read alignment with Bowtie 2. Nat. Methods 9:357-359.

Lee, J. H., Choi, J. Y., Tao, X. Y., Kim, J. S., Kim, W. and Je, Y. H. 2013. Transcriptome analysis of the small brown planthopper, Laodelphax striatellus carrying Rice stripe virus. Plant Pathol. J. 29:330-337.

Lee, K. S., Kim, S. R., Park, N. S., Kim, I., Kang, P. D., Sohn, B. H., Choi, K. H., Kang, S. W., Je, Y. H., Lee, S. M., Sohn, H. D. and Jin, B. R. 2005. Characterization of a silkworm thioredoxin peroxidase that is induced by external temperature stimulus and viral infection. Insect Biochem. Mol. Biol. 35:73-84.

Liu, B., Qin, F., Liu, W. and Wang, X. 2016. Differential proteomics profiling of the ova between healthy and Rice stripe virus-infected female insects of Laodelphax striatellus. Sci. Rep. 6:27216.

Liu, W., Gray, S., Huo, Y., Li, L., Wei, T. and Wang, X. 2015. Proteomic analysis of interaction between a plant virus and its vector insect reveals new functions of hemipteran cuticular protein. Mol. Cell. Proteomics 14:2229-2242.

Mortazavi, A., Williams, B. A., McCue, K., Schaeffer, L. and Wold, B. 2008. Mapping and quantifying mammalian transcriptomes by RNA-Seq. Nat. Methods 5:621-628.

$\mathrm{Ng}$, J. C. and Falk, B. W. 2006. Virus-vector interactions mediating nonpersistent and semipersistent transmission of plant viruses. Annu. Rev. Phytopathol. 44:183-212.

Patel, R. K. and Jain, M. 2012. NGS QC Toolkit: a toolkit for quality control of next generation sequencing data. PLoS One 7:e30619.

Pinheiro, P. V., Ghanim, M., Alexander, M., Rebelo, A. R., Santos, R. S., Orsburn, B. C., Gray, S. and Cilia, M. 2017. Host plants indirectly influence plant virus transmission by altering gut cysteine protease activity of aphid vectors. Mol. Cell. Proteomics 16(4 Suppl 1):S230-S243.
Premzl, A., Turk, V. and Kos, J. 2006. Intracellular proteolytic activity of cathepsin B is associated with capillary-like tube formation by endothelial cells in vitro. J. Cell. Biochem. 97:1230-1240.

Roberts, A. and Pachter, L. 2013. Streaming fragment assignment for real-time analysis of sequencing experiments. Nat. Methods 10:71-73.

Shiba, H., Uchida, D., Kobayashi, H. and Natori, M. 2001. Involvement of cathepsin B- and L-like proteinases in silk gland histolysis during metamorphosis of Bombyx mori. Arch. Biochem. Biophys. 390:28-34.

Sim, S., Ramirez, J. L. and Dimopoulos, G. 2012. Dengue virus infection of the Aedes aegypti salivary gland and chemosensory apparatus induces genes that modulate infection and blood-feeding behavior. PLoS Pathog. 8:e1002631.

Storr, S. J., Woolston, C. M., Zhang, Y. and Martin, S. G. 2013. Redox environment, free radical, and oxidative DNA damage. Antioxid. Redox Signal. 18:2399-2408.

Stram, Y. and Kuzntzova, L. 2006. Inhibition of viruses by RNA interference. Virus Genes 32:299-306.

Toriyama, S. 1986. Rice stripe virus: prototype of a new group of viruses that replicate in plants and insects. Microbiol. Sci. 3:347-351.

Van Rij, R. P., Saleh, M.-C., Berry, B., Foo, C., Houk, A., Antoniewski, C. and Andino, R. 2006. The RNA silencing endonuclease Argonaute 2 mediates specific antiviral immunity in Drosophila melanogaster. Genes Dev. 20:2985-2995.

Wang, X.-H., Aliyari, R., Li, W.-X., Li, H.-W., Kim, K., Carthew, R., Atkinson, P. and Ding, S.-W. 2006. RNA interference directs innate immunity against viruses in adult Drosophila. Science 312:452-454.

Wei, T.-Y., Yang, J.-G., Liao, F.-L., Gao, F.-L., Lu, L.-M., Zhang, X.-T., Li, F., Wu, Z.-J., Lin, Q.-Y., Xie, L.-H. and Lin, H.-X. 2009. Genetic diversity and population structure of rice stripe virus in China. J. Gen. Virol. 90:1025-1034.

Wu, W., Zheng, L., Chen, H., Jia, D., Li, F. and Wei, T. 2014. Nonstructural protein NS4 of Rice Stripe Virus plays a critical role in viral spread in the body of vector insects. PLoS ONE 9:e88636.

Xu, Y., Huang, L., Fu, S., Wu, J. and Zhou, X. 2012. Population diversity of rice stripe virus-derived siRNAs in three different hosts and RNAi-based antiviral immunity in Laodelphgax striatellus. PLoS ONE 7:e46238.

Yang, M., Xu, Z., Zhao, W., Liu, Q., Li, Q., Lu, L., Liu, R., Zhang, X. and Cui, F. 2018. Rice stripe virus-derived siRNAs play different regulatory roles in rice and in the insect vector Laodelphax striatellus. BMC Plant Biol. 18:219.

Yang, X.-M., Hou, L.-J., Dong, D.-J., Shao, H.-L., Wang, J.-X. and Zhao, X.-F. 2006. Cathepsin B-like proteinase is involved in the decomposition of the adult fat body of Helicoverpa armigera. Arch. Insect Biochem. Physiol. 62:1-10.

Zhao, X.-F., An, X.-M., Wang, J.-X., Dong, D.-J., Du, X.-J., Sueda, S. and Kondo, H. 2005. Expression of the Helicoverpa cathepsin b-like proteinase during embryonic development. 
Arch. Insect Biochem. Physiol. 58:39-46.

Zhao, W., Lu, L., Yang, P., Cui, N., Kang, L. and Cui, F. 2016a Organ-specific transcriptome response of the small brown planthopper toward rice stripe virus. Insect Biochem. Mol. Biol. 70:60-72.

Zhao, W., Yang, P., Kang, L. and Cui, F. 2016b. Different patho- genicities of Rice stripe virus from the insect vector and from viruliferous plants. New Phytol. 210:196-207.

Zuzarte-Luis, V., Montero, J. A., Kawakami, Y., Izpisua-Belmonte, J. C. and Hurle, J. M. 2007. Lysosomal cathepsins in embryonic programmed cell death. Dev. Biol. 301:205-217. 